\title{
MARKOV MODELS AND THE UTILIZATION OF MENTAL HEALTH SERVICES: A STUDY OF ENDOGENOUSLY DEPRESSED PATIENTS
}

\author{
DONALD L. FISHER \\ Psychology Department and Mental Healih Research Institute. The Unversity of Michigan, MI 48109, U.S.A. \\ and \\ DAVID J, KNESPER \\ Psychiatry Department and Mental Health Research Institute, The University of Michigan. MI 48109, U.S.A
}

(Received 26 May 1982)

\begin{abstract}
Patients in the mental health care system typically make more or less irregularly spaced visits to psychiatrists, both within and between episodes of a given illness. A Markov model is constructed which can predict the utilization of psychiatric services for such patients. Unlike previous Markov models of utilization, the current model takes as its staring point a model of an actual disease, specifically, endogenous depression It is shown how one can estimate the parameters of both the model of utilization and the model of depression using data which were collected for clinical research purposes. The models provide reasonable fits to the data. Several applications of the models are worked out. In addition to predicting the utilization of mental health services, the models can be used to predict incidence, prevalence and recovery rates and to predict the changes in utilization which parallel changes in treatment regimens.
\end{abstract}

Prediction of the utilization of medical services has become increasingly important. In this paper a model of utilization is constructed which can be used to predict the use of both outpatient and inpatient services by endogenously depressed patients. The model is influenced by the incidence. prevalence and recovery rates of depression and the severity of the disease at each moment in time. Some attempt is made at the end of the paper to show how one might incorporate into the model of utilization various of the economic factors which have been shown to have an affect on utilization (a detailed discussion of the economic factors can be found in [1]).

Note that from the standpoint of utilization, depression is an important disease to consider. It is widely spread throughout major portions of the population. And recently there have come into existence therapeutic regimens which have the potential for greatly increasing the recovery rates of endogenously depressed patients[2]. It is of some consequence to be able to predict the effect that changes in these rates will have on utilization.

The literature on health services planning is extensive (for a general review see [1]), in part because of the variety of existing services offered by the medical profession and in part because of the many different categories of patients which must be considered. In certain cases it has been possible to construct detailed mathematical models of utilization. A systematic review of these models will not be attempted here since the number of different types of such models which have been used for medical planning is itself quite large (e.g. see recent work by Kao [3-5] and Sullivan and Blair [6]). This paper will confine itself to Markov models. Markov models have been used extensively in medical planning on both a system wide basis (e.g. [7-10]) and within more restricted health contexts $(\mathrm{e} . g .[5,11-17])$. Only recently have Markov models been used for mental health plan- ning. Specifically, Sweillam and Tardiff[18] employed Markov models to predict personnel requirements for inpatient psychiatric services. This paper will focus on the development of Markov models which can be used for both inpatient and outpatient services.

The problem of health services planning is particularly difficult when the disease being considered is endogenous depression or one similar to it, i.e. when patients' needs are irregularly spaced over reasonably long periods of time [19-22]. In fact, no models of utilization have been constructed to date which explicitly take account of a protracted disease process. Models of utilization specific to long term disorders have not been developed if only because related models of the underlying disease processes are difficult to construct and test. Such models generally require data from a prospective longitudinal survey of a geographically stable, non-hospitalized population, data which until now have not been collected[23]. However, acceptable alternatives to a longitudinal survey exist in certain cases. One such alternative is explored in this study. Once a working model of the disease process is constructed, it is then possible to consider a model of utilization which is sensitive to the disease process.

In addition to requiring a model of the disease process, a model of utilization developed for patients in the mental health care context will require a more detailed specification of the state space than has been considered to date. Note that the Markov models constructed to date have considered one and the same (for purposes of prediction) a patient's state of health and a patient's utilization of services: very sick patients required extensive services, moderately sick patients required relatively few services, and so on. The extension of the mental health planning context to outpatients as well as inpatients requires a separation of the state of health and the level of personnel utilization when it is desirable to use a small unit time interval such as the day. Note that a 
very sick patient may or may not make a demand on the time of mental health personnel on a given day. For example. a sick patient not in the hospital and currently receiving some form of treatment makes only periodic demands on a psychiatrist's time, perhaps once every two weeks for an hour or so whereas a patient who is in the hospital uses various forms of psychiatric services throughout the day every day he or she remains an inpatient. The approach used to deal with the separation of states of illness and levels of utilization will depend greatly on the particular illness being considered. In this paper, as noted above, only one illness will be examined, specifically, endogenous depression.

One final problem presents itself before a model of utilization can actually be tested. In particular, it must be possible to estimate the model parameters. This is not always an easy process, especially when the more convenient parameter estimation procedures require knowledge of the level of daily functioning for each patient. Unfortunately, information is typically collected from each patient only when the patient actually makes a visit to an outpatient clinic. Thus large, sporadic gaps exist in the data. Since it is expensive to gather daily data from outpatients, it would be nice if one could use the clinical data in its current form. Methods are described in this paper which permit the investigator to obtain the parameter estimates from the existing clinical data.

In summary, if one wants to predict the utilization of psychiatric services by endogenously depressed patients, then there are three problems which must be overcome. First, a model of depression which approximates the disease process must be constructed. Second, a model of utilization must be developed which at one and the same time is kept separate from the model of depression (since depressed outpatients do not make a visit every day) and is dependent on it (since the more depressed patients make more frequent visits). Finally, a way of estimating model parameters from clinical data must be devised Once the models are developed and the parameters estimated, it is then possible to test the fit of the models to the data.

\section{MODEL OF DEPRESSION}

In this section a model will be constructed which can be used to predict the disease course of endogenously depressed patients. Endogenous depression is an illness which frequently recurs over the course of a lifetime, which is more severe at some times than others, and which is not closely correlated with events in a patient's history [24]. The patients used in this study were in a program of intensive therapy. While the model constructed in this section will be referred to as simply a model of depression, it should be kept in mind that the model extends only to endogenously depressed patients receiving intensive therapy. The model of depression will be used to predict a patient's state $s_{t}$ of depression on day $t_{k}$, given knowledge of the patient's state of depression $s$, at some point earlier in time.

Recently, several different stochastic models of depression have been developed [23]. These models are used to predict the incidence, prevalence and recovery rates of patients diagnosed as depressed (note that no distinction was made between endogenous depression and other forms of depression). The models used the year as the unit of time and used only two states of depression, depressed and not depressed. It is important to note that the Markov property did not obtain for the patients in the study. That is, the probability that a patient was depressed in year $y_{t}$ depended not only on a patient's state of depression in year $y_{t-1}$, but also on a patient's state of depression at least five years previous. This finding does not argue against the use of a Markov process to model endogenous depression since the patient sample in the Dunn and Skuse [23] study probably included both exogenously depressed individuals (individuals whose depression can be traced to changing circumstances in their personal lives) as well as endogenously depressed patients.

Four assumptions about the behavior of endogenously depressed patients define the stochastic model described in this section. First, it is assumed that patients can be placed into one of seven mutually exclusive and exhaustive states (these states are defined in the Method Section). Second, it is assumed that subjects make at most one transition between states over the course of a single day. Third, it is assumed that the probability that a patient makes a transition to state $s_{1}$ on day $t_{k}$ depends on only the state of the individual on the immediately preceding day $t_{k-1}$. Finally, it is assumed that the probability that a patient makes a transition from state $s_{s}$ to state $s_{k}$ does not depend on the day the patient makes the transition. Together, these assumptions define the stochastic model as a stationary, finite state, discrete time, first order Markov process, or more simply, as a Markov chain.

It is well known that knowledge of the transition probabilities $p_{i j}$ between states $s_{1}$ and $s_{1}$ and knowledge of the entering state of a patient allows one to predict the probability that a patient is in a given state on any specified day in the future. More generally, if one knows the transition probabilities and the number $n_{i}\left(t_{0}\right)$ of patients in each state $s_{1}$ on the first or entering day $t_{0}$ then one can predict the number $n_{f}\left(t_{k}\right)$ of patients in each

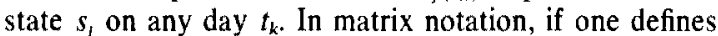
the entering status vector $N\left(t_{0}\right)$ as consisting of entries $n_{i}\left(t_{0}\right)$, then the status vector $N\left(t_{k}\right)$ for day $t_{k}$ can be obtained from the matrix $P$ of transition probabilities as follows:

$$
N\left(t_{k}\right)=N\left(t_{0}\right) P^{k}
$$

As is evident from eqn (1), the matrix $P$ of transition probabilities figures critically in the predictions. Not all transitions between states $s_{z}$ and $s_{i}$ are allowed in the model. The characteristics of depression suggest that only certain transitions will be critical, i.e. will have probabilities significantly greater than zero. These transitions are indicated by the presence of a positive integer in Fig. 1. Transitions which are labelled with identical integers (e.g. transitions from states $s_{2}, s_{3}$ and $s_{4}$ to $s_{6}$ ) are assumed to have the same probability of occurrence.

In order to arrive at actual predictions and compare the predictions with observed data, it is necessary first to establish rules for placing patients into the various states of depression and then actually to estimate the transition probabilities.

\section{Method}

Subjects. Individuals in the sample of 77 patients used in this study were all diagnosed as endogenously depressed. Most were given antidepressant medications. Some were also seen in psychotherapy. The earliest records go back to 1974. The latest records used extend through September 1980. 


\begin{tabular}{|c|c|c|c|c|c|c|c|c|}
\hline & & \multicolumn{7}{|c|}{ State on Day $t_{k+1}$} \\
\hline & & $\mathbf{s}_{1}$ & $s_{2}$ & $\mathbf{s}_{3}$ & $\mathrm{~s}_{4}$ & $s_{5}$ & $s_{6}$ & $s_{7}$ \\
\hline \multirow[b]{3}{*}{ State } & \multirow[t]{2}{*}{$s_{1}$} & 1 & 2 & 0 & 0 & 0 & 0 & 3 \\
\hline & & 4 & 5 & 6 & 0 & 0 & 7 & 0 \\
\hline & $s_{3}$ & 0 & 8 & 9 & 10 & 11 & 7 & 0 \\
\hline On & $s_{4}$ & 0 & 0 & 12 & 13 & 14 & 7 & 0 \\
\hline \multirow[t]{3}{*}{ vay $t_{k}$} & $\mathbf{s}_{5}$ & 15 & 0 & 0 & 0 & 16 & 17 & 0 \\
\hline & \multirow[t]{2}{*}{$s_{6}$} & 18 & 0 & 0 & 0 & 0 & 19 & 0 \\
\hline & & 18 & 0 & 0 & 0 & 0 & 0 & 19 \\
\hline
\end{tabular}

Note: a positive integer in a cell indicates a first order transition is permitted; a zero in a cell indicates that a first order transition is not permitted.

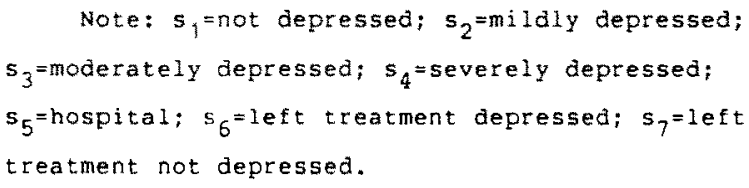

Fig. 1. Permissible first order transitions in the model of depression.

States of depression. Subjects were placed in state $s_{1}$ (not depressed), $s_{2}$ (mildly depressed), $s_{3}$ (moderately depressed) or $s_{4}$ (very depressed) for a given day on the basis of their score on the Carroll Depression Rating, a self-administered form of the Hamilton Depression Rating[25]. Specifically, the following correspondence was made between states of depression and scale ratings: state $s_{1}$ was recorded if the scores on the Carroll Depression Rating were in the range 1-7 inclusive; state $s_{2}$ was recorded if the scores were in the range 8-12; state $s_{3}$ was recorded if the scores were in the range 13-19; and state $s_{4}$ was recorded if the scores were in the range 20-52. Patients in the hospital were placed in state $s_{s}$. Finally, two states of termination were defined. Patients were placed in state $s_{6}$ if they were depressed (i.e. in states $s_{2}$ through $s_{5}$ ) when they last made a visit while patients were placed in state $s_{7}$ if they were not depressed (i.e. in state $s_{1}$ ) when they last made a visit.

Parameter estimation. Figure 1 makes clear the 19 unique transition probabilities which required estimation. A four step procedure was used, a different step for four mutually exclusive and exhaustive subsets of the complete set of 19 transition probabilities. The four step procedure is needed because the data were collected from outpatients only on those days a visit to a psychiatrist was made. Therefore, the data reflect the great heterogeneity in the spacing of visits. Throughout the discussion of parameter estimation it will be important to keep in mind the distinction between a true matrix $M$ of transition probabilities and an estimated matrix $\mathscr{H}$ of transition probabilities which is made optimal according to some criterion (more generally, italics will be used to indicate any estimate which is being optimized). Unless otherwise indicated, a least squares criterion was used as the optimizing criterion. A least squares criterion selects as parameter estimates those values of the parameters which minimize the sum of the squares of the differences between observed and predicted scores.

In step 1 only those cells in the upper left hand 4 by 4 submatrix of Fig. 1 were examined. The transition probabilities $p_{2}$ in these cells give the probability that a patient in states $s_{1}$ through $s_{4}$ will make a transition on the very next day to one of the first four states. The clinical data are in such a form that rather than estimating the transition probabilities directly, it is easiest to estimate what will be referred to as modified transition probabilities $q_{t r}$ A modified transition probability $q_{t,}$ is the probability that a patient will be in state $s,(j=1,2,3$, 4) on day $t_{k+1}$ given that the patient was in state $s_{1}(1=1$, $2,3,4)$ on day $t_{k}$ and given that the patient did not enter any one of states $s_{5}, s_{6}$ or $s_{7}$ on day $t_{k+1}$. This means that the modified transition probabilities sum to one across the first four columns, i.e.

$$
\sum_{i=1}^{4} q_{1 j}=1 \text { for } i=1,2,3,4 .
$$

These modified estimates are corrected in a later step (see step 3 and Appendix A), i.e. it is shown how to compute $p_{i t}$ from the corresponding $q_{v i}$.

Estimates of the matrix $Q$ of true modified transition probabilities were obtained as follows. Let $V(k)$ and $W_{i}(k)$ be 1 by 4 row vectors. The entry $v_{i}(k)$ in vector $V(k)$ represents the number of patients who have made recorded visits (recorded visits are visits in which the Carroll Depression Rating was administered; this rating 
was not administered on all visits) on days $t_{r}$ and $t_{r+k}$, who were in state $s_{t}$ on day $t_{r}$, and who did not move to states $s_{5}, s_{6}$ or $s_{7}$ sometime between days $t_{r}$ and $t_{r+k}$. The entry $w_{i j}(k)$ in vector $W_{i}(k)$ represents the number of patients who have made recorded visits on days $t_{r}$ and $t_{r+k}$, who were in state $s_{t}$ on day $t_{r}$ who were in state $s_{1}$ on day $t_{r+h}$, and who did not move to states $s_{5}, s_{6}$ or $s_{7}$ sometime between days $t_{r}$ and $t_{r+h}$.

A prediction of the quantity $k_{i j}^{\prime}(k)$ can be obtained if one makes two simplifying assumptions. In order to motivate these assumptions, note that $\boldsymbol{w}_{1 j}(k)$ can be written as the product:

$$
\begin{aligned}
w_{t u}(k)= & v_{t}(k) P\left(S_{r+h}=j, V_{r+h}=1\right. \\
& \left.S_{r}=i, V_{r}=1, V_{r+h}=1, B=1\right),
\end{aligned}
$$

$$
=v_{t}(k) P(i, j, r, k) \text {. }
$$

where $S_{r}$ is a random variable set equal to the state of a patient on day $t_{r}$; where $V_{r}$ is an indicator random variable set equal to 1 if the patient is visiting a therapist on day $t$, and is equal to 0 otherwise; and where the indicator random variable $B$ equals 1 if the patient does not enter any one of states $s_{\varsigma}, s_{6}$ or $s_{7}$ in the days intervening between the two visits. Let $C$ be the event $\left\{S_{r}=i, V_{r}=1, V_{r / k}=1, B=1\right\}$. Then the above conditional probability $P(i, j, r, k)$ can be rewritten as the sum of the product of three conditional probabilities:

$$
\begin{gathered}
\sum_{m=1}^{4} \sum_{n=1}^{4} \sum_{u=0}^{1} \sum_{b=0}^{1} P\left(S_{r+h}=j, V_{r+h}=1 \mid S_{r+h} \quad=n,\right. \\
\left.V_{r+h-1}=a, S_{r+1}=m, V_{r+1}=b, C\right) \\
P\left(S_{r+h-1}=n, V_{r+h \quad 1}=a \mid S_{r+1}=m, V_{r+1}\right. \\
P\left(S_{r+1}=m, V_{r+1}=b \mid C\right) .
\end{gathered}
$$

The assumptions one must make can now be made clear. First, assume that knowledge that a patient has made or will make a visit to a psychiatrist does not change the course of endogenous depression. In particular assume that the conditional probability $P\left(S_{d}=\right.$ $s_{i} \mid S_{d e_{e}}=s_{1}, \ldots, S_{d-f}=s_{h}, V_{d+e}=a, \ldots, V_{d h}=b, B=$ 1) can be rewritten as $P\left(S_{d}=s_{t} \mid S_{d}{ }_{c}=s_{j}, \ldots, S_{d}=s_{k}\right.$, $B=1$ ). This assumption seems reasonable enough when the depression is endogenous. Visits to a therapist in this case serve other purposes, e.g. monitoring physical health, keeping track of responses to drug therapy, etc.

Second, assume that the random variables $S_{r}$ and $V_{r}$ are conditionally independent so that $P\left(S_{d}=s_{l}, V_{d}=\right.$ $c \mid S_{d-e}=s_{j}, \ldots, S_{d-f}=s_{k}, V_{d+g}=a, \ldots, V_{d h}=b, B=$ 1) can be rewritten as the product of two probabilities: $P\left(S_{d}=s_{t} \mid S_{d e}=s_{1} \ldots, S_{d j}=s_{h}, V_{d+g}=a, \ldots, V_{d h}=\right.$ $b, B=1)$ and $P\left(V_{d}=c \mid S_{d-e}=s_{\eta}, \ldots, S_{d-f}=s_{k}, V_{d+g}=\right.$ $\left.a, \ldots, V_{d-h}=b, B=1\right)$. Note that by the assumption described in the previous paragraph the first term in the product reduces to $P\left(S_{d}=s_{1} \mid S_{d-e}=s_{j}, \ldots, S_{d-f}=s_{k}\right.$, $B=1$ ). This in turn can be written more simply as the multistep transition probability $q_{n}(e)=$ $P\left(S_{d}=s_{1} \mid S_{d} e^{\prime}=s_{1}, B=1\right)$ by virtue of the Markov assumption in the model of depression, or if $e=1$, as $q_{\mu}$. Also note that when summing over $a, b=1,2$ in eqn (4) the second probability in the above product equals 1 .
Finally, it should be mentioned that the second assumption is reasonable if the great majority of visits are scheduled by the psychiatrist ahead of time (an assumption which, empirically, has much support).

In short, if the above two assumptions are met, then one can predict the quantity $w_{i j}(k)$ from the relation,

$$
\mathscr{W}_{1}(k)=v_{1}(k) \sum_{m-1}^{1} \sum_{n-1}^{1} q_{n j} q_{m n}(k-2) q_{1 m}
$$

where $q_{m n}(k-2)$ is the conditional multistep transition probability (i.e. the probability that a patient is in state $s_{n}$, given that the patient was in state $s_{m} k-2$ days previous). In matrix notation, Equation (5) can be rewritten as:

$$
W_{i j}(k)=v_{t}(k) E_{i}^{\prime}[9]^{k} E_{j}
$$

where $E_{t}^{\prime}$ is a row vector with a 1 in the $i$ th column and 0 's everywhere else and where $E$, is a column vector with a 1 in the $j$ th row and 0 's everywhere else.

A program was used[26] which then minimized the sum $y$ of squared differences between observations $\mathcal{W}_{i j}(k)$ and predictions $\mathscr{W}_{i j}(k)$ over all intervisit intervals up to and including a 49 day intervisit interval:

$$
\begin{aligned}
\min (y)= & \min \left[\sum_{k=1}^{4 y} \sum_{1}^{4} \sum_{j}^{4}\right. \\
& \left.\left(w_{15}(k)-w_{1,}(k)\right)^{2}\right] .
\end{aligned}
$$

Step 2 involves the estimation of transition probabilities from state $s_{5}$ to states $s_{1}, s_{5}$ and $s_{6}$ (numbers 15 , 16 and 17 in Fig. 1). Since data for every such transition is available, it is possible to estimate these using simple frequency counts or proportions.

In step 3 the transition probabilities from states $s_{1}$ through $s_{4}$ to states $s_{6}$ and $s_{7}$ and from states $s_{3}$ and $s_{4}$ to $s_{5}$ are estimated (numbers 3, 7,11 and 14 in Fig. 1). Note that neither of the procedures outlined in the above two steps can be used to estimate the present set of transition probabilities (see Appendix B). Nor can the present procedure be used to estimate the transition probabilities in steps 1 and 2 . Instead, the current step uses the mean first passage times from state $s_{t}$ to state $s_{\text {j. }}$. The mean first passage time from state $s_{i}$ to state $s_{1}$ is defined as the expected number $f_{11}$ of days it takes the patient to reach state $s_{f}$, given that the patient starts in state $s_{\imath}$ on day $t_{0}$. Note that when computing the mean first passage times, it was assumed that the transition probabilities from states $s_{6}$ and $s_{7}$ to state $s_{1}$ were equal to one (see discussion of step 4 below).

The program by Becker [26] was again used to obtain the least squares estimates of the parameters:

$$
\begin{aligned}
& \min (\mathscr{y})=\min \left[\sum_{i=1}^{5} \sum_{j=6}^{7}\left(\mathscr{F}_{1 j}-f_{1 j}\right)^{2}\right. \\
& 1 \sum_{i=3}^{4}\left(\begin{array}{ll}
\mathscr{F}_{15} & \left.f_{t 5}\right)^{2}
\end{array}\right] \text {. }
\end{aligned}
$$

Note that $F_{1}$ is an estimate of the expected first passage time derived from the 7 by 7 matrix $P$ (see Appendix A) of transition probabilities while $f_{i j}$ is computed directly from the data:

$$
f_{11}=\sum_{k-1}^{n} x_{i j k} / n
$$


where $n$ is the number of individuals starting in state $s_{i}$ and terminating in state $s_{\mathrm{J}}$ and where $x_{i, k}$ is the number of days intervening between entries to the two states for patient $k$.

The fourth and final step requires estimating the transition probabilities from state $s_{6}$ to states $s_{1}$ and $s_{6}$ and from state $s_{7}$ to states $s_{1}$ and $s_{7}$ (numbers 18 and 19 in Fig. 1). Unfortunately, there was not enough information to form reliable estimates. In the absence of this information, it seems reasonable in the current context to set the transition probabilities from states $s_{6}$ and $s_{7}$ to state $s_{1}$ equal to one. This seems reasonable for the transition probability from state $s_{7}$ to $s_{1}$ since, by definition of state $s_{7}$, a patient had been in state $s_{1}$ on the last visit. And this seems reasonable for the transition probability from state $s_{6}$ to $s_{1}$ since, although the patient was not in state $s_{1}$ on the last visit, it is known that the patient has not made a visit to a psychiatrist for some period of time and therefore, is presumably not sick. Note that if these assumptions are seriously violated, then the agreement between the predicted and observed first passage times in step 3 will not be good.

\section{Results}

The least squares estimate $\mathscr{P}$ of the matrix of transition probabilities is displayed in Fig. 2. The estimates of the transition probabilities make good clinical sense, indicating at a very broad level that the multistep parameter estimation procedure is not distorting the true probabilities beyond all recognition. For example, note that the probability of making a transition from being very depressed as an outpatient (state $s_{4}$ ) to the hospital (state $s_{5}$ ) is relatively high and clearly greater than the probability of making the same transition from a state of only moderate depression (

More quantitative indications of fit can be provided. As previously explained, the estimated matrix 2 of modified transition probabilities can be used to predict the number $W_{t \jmath}(k)$ of patients who made visits on days $t_{r}$ and $t_{r+k}$, who were in state $s_{1}$ on day $t_{r+k}$, who were in state $s_{2}$ on day $t_{5}$ and who did not visit states $s_{5}, s_{6}$ or $s_{7}$ on any intervening days. The matrix 2 is displayed in Fig. 3 while Fig. 4 presents the observed $w_{i j}(49)$ and predicted $\mathscr{W}_{y \jmath}(49)$ quantities for an intervisit interval of

\begin{tabular}{|c|c|c|c|c|c|c|c|c|}
\hline & & \multicolumn{7}{|c|}{ State on Day ${ }^{t} k+1$} \\
\hline & & $s_{1}$ & $\mathbf{s}_{2}$ & $s_{3}$ & $s_{4}$ & $s_{5}$ & $s_{6}$ & $s_{7}$ \\
\hline \multirow[b]{3}{*}{ Stace } & $\mathbf{s}_{1}$ & .993 & .005 & 0.0 & 0.0 & 0.0 & 0.0 & .002 \\
\hline & $\mathbf{s}_{2}$ & .030 & .776 & .052 & 0.0 & 0.0 & .141 & 0.0 \\
\hline & $\mathbf{s}_{3}$ & 0.0 & .027 & .433 & .026 & .373 & .141 & 0.0 \\
\hline on & $s_{4}$ & 0.0 & 0.0 & .002 & .058 & .799 & .141 & 0.0 \\
\hline \multirow[t]{3}{*}{ Day $t_{k}$} & $\mathbf{s}_{5}$ & .019 & 0.0 & 0.0 & 0.0 & .979 & .001 & 0.0 \\
\hline & $s_{6}$ & 1.0 & 0.0 & 0.0 & 0.0 & 0.0 & 0.0 & 0.0 \\
\hline & $s_{7}$ & 1.0 & 0.0 & 0.0 & 0.0 & 0.0 & 0.0 & 0.0 \\
\hline
\end{tabular}

Fig. 2. Matrix $\mathscr{P}$ of predicted first order transition probabilities.

\begin{tabular}{|c|c|c|c|c|c|}
\hline & & \multicolumn{4}{|c|}{ State on Day $t_{k+1}$} \\
\hline & & $\mathbf{s}_{1}$ & $\$_{2}$ & $s_{3}$ & $\mathbf{s}_{4}$ \\
\hline \multirow{4}{*}{$\begin{array}{l}\text { State on } \\
\text { Day } t_{k}\end{array}$} & \multirow{2}{*}{$\begin{array}{l}s_{1} \\
s_{2}\end{array}$} & .995 & .005 & 0.0 & 0.0 \\
\hline & & .035 & .904 & .064 & 0.0 \\
\hline & \multirow[t]{2}{*}{$s_{3}$} & 0.0 & .055 & .891 & .046 \\
\hline & & 0.0 & 0.0 & .033 & .968 \\
\hline
\end{tabular}

Note: the transition probabilities within a row will

not always sum exactiy to one because of rounding errors.

a It is shown in the text and Appendix A how to

obtain the matrix $Q$ from the matrix $P$.

Fig. 3. The matrix 2 of modifed predicted first order transition probabilities for depressed patients. 


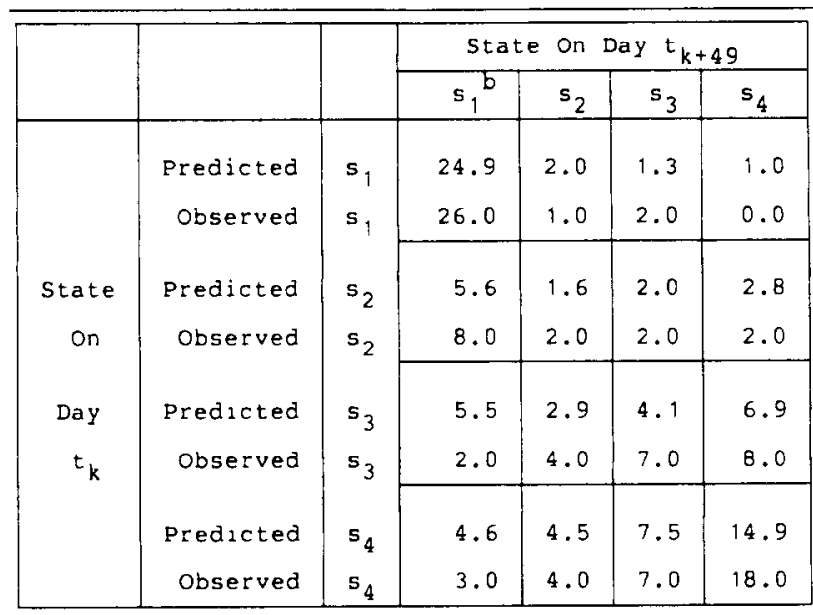

a Note that patients qualified as having made

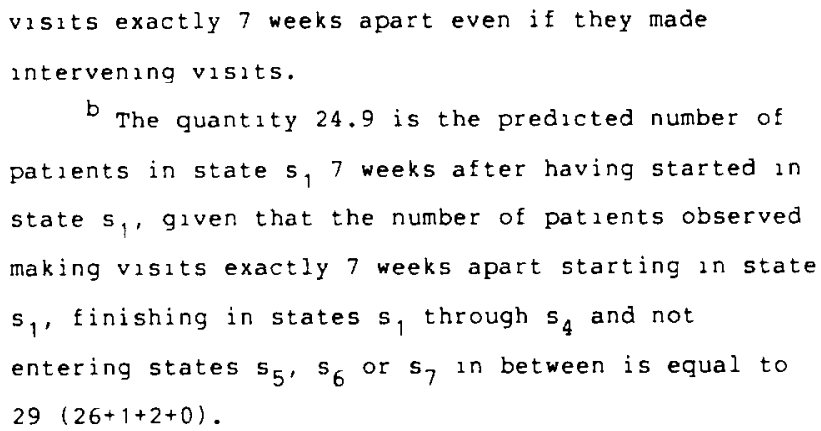

Fig. 4. The predicted and observed number of patients making visits separated by 7 weeks ${ }^{\text {t }}$ to states $s_{i}$ and $s_{,}$.

49 days. Eleven out of the sixteen predictions differ from the observations by less than 2 . No difference is greater than 4. The root mean square deviation for visits separated by exactly seven weeks is only 1.78 .

A comparison of observed and predicted mean first passage times can also give an indication of fit. Figure 5 shows that there is a very close agreement between the observations and predictions (see transitions from state $s_{3}$ to state $s_{5}$, and from states $s_{4}$ and $s_{5}$ to states $s_{5}, s_{6}$ and $s_{7}$ ). The exceedingly long expected first passage times to state $s_{4}$ reflect the fact that many patients go directly from state $s_{3}$ to the hospital (thereby bypassing state $s_{4}$ as an outpatient) and that very depressed patients go almost immediately to the hospital.

\section{Discussion}

The Markov model of depression described in this section can be used to predict the number of individuals in a particular state of depression at some point in the future. The model can also be used to predict the mean first passage time from one state to another. The agreement between the various predictions and the observations is encouraging, especially given the need for a multistep parameter estimation procedure. It should be emphasized that the relative success of the multistep parameter estimation procedure has important practical applications. In particular, clinicians can continue to collect data on a visit only basis. Such data are sufficient for current modelling and therefore increase the potential use of the models in applied settings.
Unfortunately, there was no easy way to perform statistical tests of the model since information on a patient's state of illness was not recorded every day (had such information been recorded, one could have formed maximum likelihood estimates of the parameters and then tested the estimates against various hypotheses). Ideally, one would like to know whether the rather counterintuitive stationarity and memoryless assumptions hold up in practice. More theoretical studies of the disease process would presumably want to gather the needed additional observations.

The computation of incidence, prevalence and recovery rates can easily be determined from the matrix $\mathscr{P}$. Suppose a person is defined as beginning an episode of depression if, starting in states $s_{1}, s_{2}$ or $s_{7}$, the individual passes to states $s_{3}, s_{4}, s_{5}$ or $s_{6}$ by the end of the week. Make states $s_{3}$ through $s_{6}$ absorbing. Then the incidence rate $R_{\boldsymbol{r}}$ for a week beginning on day $t_{r}$ and having entering status vector $N\left(t_{0}\right)$ can be written as:

$$
\begin{gathered}
R_{i}=\sum_{j \in C} \sum_{k=3}^{6} a_{j h}(7) n_{j}\left(t_{r}\right), \\
C=\{1,2,7\},
\end{gathered}
$$

where $a_{\jmath k}(7)$ is the probability that an individual in state $s_{1}$ on day $t_{r}$ is in state $s_{k}$ on day $t_{r+7}$. This probability is computed using matrix $\mathscr{P}$ when states $s_{3}$ through $s_{6}$ are made absorbing. The quantity $n_{j}\left(t_{r}\right)$ is computed using eqn (1) and the entering status vector $N\left(t_{0}\right)$.

The recovery rate $R_{r}$ for a week beginning on day $t_{r}$ 


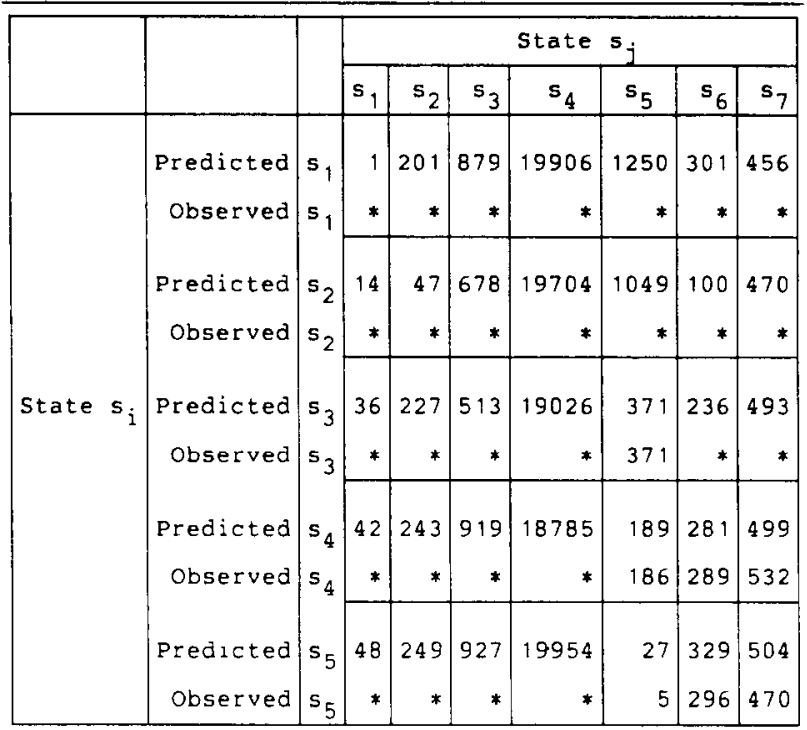

Note: entry designated by "*" indicates that

there were no observations.

Fig. 5. Matrix of predicted and observed mean first passage times from state $s_{t}$ to state $s_{r}$

and having entering status vector $N\left(t_{0}\right)$ is computed similarly.

$$
\begin{gathered}
R_{r}=\sum_{j \in C} \sum_{k=3}^{6} b_{k j}(7) n_{k}\left(t_{r}\right), \\
C=\{1,2,7\} .
\end{gathered}
$$

The quantity $b_{k}(7)$ is the multistep transition probability computed using matrix $\mathscr{P}$ when $s_{1}$ and $s_{2}$ are made absorbing. Note that it is assumed, as above, that a patient is depressed if in states $s_{3}$ through $s_{6}$ and not depressed if in states $s_{1}, s_{2}$ or $s_{7}$.

Finally, the prevalence rate for day $t_{r}$ given entering status vector $N\left(t_{0}\right)$ is simply the sum,

$$
\sum_{j=3}^{6} n_{j}\left(t_{r}\right)
$$

i.e. it is simply the number of patients in the more depressed states.

\section{MODEL OF UTILIZATION}

Given that a suitable model of the disease process has been constructed it is then possible to construct a model of utilization which is tied to the model of the disease process. It needs to be emphasized that, by itself, the model of depression cannot be used to predict the number of mental health personnel required for the treatment of a given sample of patients. As noted in the introduction, knowledge that a patient is, say, very depressed on day $t_{r}$ does not indicate whether the patient will or will not be receiving treatment on that day. What is needed is a model that predicts the number of patients receiving treatment on a particular day in a particular state. One can then use the predicted number of patients reciving treatment to obtain the predicted use of mental health personnel. In this section a Markov model of utilization is developed from which one can obtain both of the above predictions. It should be noted that the Markov model of utilization is formally identical to the Markov model of depression, i.e. both are Markov chains.

Throughout most of this section attention will be focussed on the development of the model of utilization to the point where it can make the first type of prediction referred to above, that is, to the point where it can predict the probability that a patient will be making a visit to a therapist in a particular state on a given day. Since visits are generally in terms of integer multiples of weeks, it would be easiest to use the week as the basic unit of time in the Markov model. However, this would do some injustice to the data since the patients in the most depressed state frequently make visits separated by only a couple of days. Thus the day was kept as the basic time unit.

The model of utilization differs from the model of depression in the assignment of outpatient states. Specifically, outpatient states in the model of utilization identify not only the severity of a patient's depression but also (a) whether the patient is or is not visiting a therapist and (b) whether the patient made a visit exactly $k$ weeks previous, $k$ weeks and 1 day previous, $k$ weeks and 2 days previous, etc. Expanding the outpatients states in this fashion makes it possible to model the observation that visits are spaced exactly an integer number of weeks apart for mildly or moderately depressed patients while visits are much more closely spaced for very depressed patients.

More concretely, each of states $s_{1}$ through $s_{4}$ in the model of depression becomes one of eight states in the model of utilization. The eight states are listed in Fig. 6 down the left hand column: states $s_{t v}$ and states $s_{t 1}$ through $s_{7}$. Outpatients in state $s_{1}$ of the model of depression making a visit are placed in state $s_{v}$. Having made a visit, outpatients can either make another visit on the very next day (in which case they go to state $s_{r v}$, $s_{1-1, v}$ or $s_{t+1, v}$ ) or not make a visit, in which case they go 


\begin{tabular}{|c|c|c|c|c|c|c|c|}
\hline & & \multicolumn{6}{|c|}{ State on Day $t_{k+1}$} \\
\hline \multirow[b]{3}{*}{ State } & $s_{1 v}$ & $s_{1 V}$ & $s_{1-1, v}$ & $\mathbf{s}_{i+1, v}$ & $s_{12}$ & $s_{1-1,2}$ & $s_{2+1,2}$ \\
\hline & $s_{i 1}$ & $s_{i v}$ & $\mathbf{s}_{\mathbf{i}-1, v}$ & $s_{i+1}, v$ & $s_{i 2}$ & $s_{i-1,2}$ & $\mathbf{s}_{1+1,2}$ \\
\hline & $s_{12}$ & $\mathrm{~s}_{1 \mathrm{~V}}$ & $s_{1}-1, v$ & $\mathbf{s}_{1}+1, \mathrm{v}$ & $s_{13}$ & $s_{1}-1,3$ & $s_{2}+1,3$ \\
\hline On & $\mathbf{s}_{i 3}$ & $\mathrm{~s}_{\mathrm{iv}}$ & $s_{1}-1, v$ & $\mathrm{~s}_{1}+1, \mathrm{v}$ & $s_{i 4}$ & $\mathbf{s}_{i-1,4}$ & $s_{i+1,4}$ \\
\hline Day & $s_{14}$ & $\mathrm{~s}_{1 \mathrm{~V}}$ & $s_{1}-1, v$ & $s_{1+1, v}$ & $s_{15}$ & $s_{i-1,5}$ & $\mathbf{s}_{i+1,5}$ \\
\hline \multirow[t]{3}{*}{$\mathrm{T}_{\mathrm{k}}$} & $s_{i 5}$ & $\mathrm{~s}_{1 \mathrm{~V}}$ & $s_{1-1, v}$ & $s_{1}+1, v$ & $s_{i 6}$ & $s_{1}-1,6$ & $\mathbf{s}_{i+1,6}$ \\
\hline & $\mathbf{s}_{i 6}$ & $s_{i v}$ & $s_{i-1, v}$ & $s_{2}+1, v$ & $s_{17}$ & $s_{1-1,7}$ & $\mathbf{s}_{1+1,7}$ \\
\hline & $s_{17}$ & $s_{1 v}$ & $5,1,0$ & $s_{1}+1, v$ & $s_{i 1}$ & $s_{i-1,1}$ & $\mathbf{s}_{1+1,1}$ \\
\hline
\end{tabular}

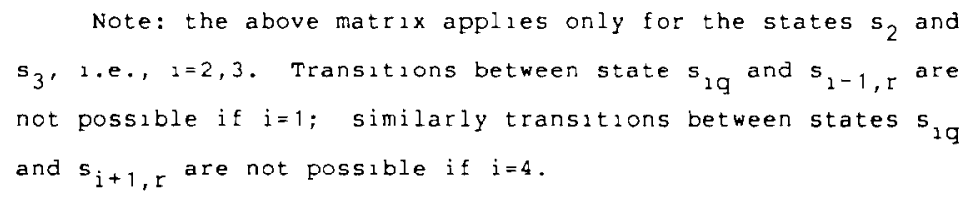

Fig. 6. One day transitions in model of utilization.

to state $s_{i 2}, s_{i-1,2}$ or $s_{t+1,2}$. Thus, for example, a pattent who remained in state $s_{1}$ and who made visits on the first and fourth days would have entered states $s_{1 \mathrm{r}}, s_{12}, s_{13}$ and $s_{1 v}$. Alternatively, a patient who made visits on the first and tenth days would have entered states $s_{1 v}, s_{12}$. $s_{13}, s_{14}, s_{14}, s_{141}, s_{17}, s_{11}, s_{12}, s_{1,}$. Finally, a patient who remained in state $s_{1}$ and who made visits on the first, third and sixth days would have entered states $s_{12}, s_{12}$, $s_{1 v}, s_{12}, s_{13}$ and $s_{1 v}$.

As noted above, the model has the virtue of being able to mimic the predominance of weekly visits for moderately depressed patients while still allowing more closely spaced visits for very depressed patients. This is easily accomplished by making the probability $p_{w}$ that a moderately depressed patient in one of states $s_{t v}, s_{t 1}, \ldots$, $s_{16}$ on day $t_{r}$ visits a therapist in state $s_{f v}$ on day $t_{r+1}$ very small and by making the probability $q_{1 v}$ that patient in state $s_{t}>$ on day $t_{r}$ visits a therapist in state $s_{v}$ on day $t_{r+1}$ relatively high. Thus, it will typically take $7 k(k \geq 7, k$ an integer) days for a moderately depressed patient to make a return visit. For more severely depressed patients one can simply increase the probability $p_{i v}$ that the patient in one of states $s_{1 v}, s_{11}, \ldots, s_{16}$ will make a visit to a therapist in state $s_{j v}$ on the very next day. The reader will note that the above discussion assumes that the probability $p_{v v}$ of going from states $s_{1 v}, s_{t 1}, \ldots, s_{16}$ to state $s_{j v}$ does not vary with the day. A more complex model would need to consider possible variations in this parameter.

Finally, note that the complete model of utilization consists of 35 states: the 32 states associated with states $s_{1}$ through $s_{4}$ in the model of depression ( 8 states of utilization for each of the 4 states of depression) plus states $s_{5}, s_{6}$ and $s_{7}$ (these states are identical in the models of utilization and depression).

\section{Method}

Subjects. The same patients were used to fit the models of depression and utilization.

Parameter estimation. There are a total of 8 parameters to estimate, two ( $p_{v}$ and $q_{t v}$ ) for each of the four states of depression $s_{1}$ through $s_{4}$. Suppose that it is currently day $t_{r-1}$ and that as of tomorrow the patient will have made a visit an integral number of weeks previous, i.e. the patient is in state $s_{17}$. The probability that the patient makes a transition to state $s_{v v}$ will be defined as the product of probabilities $p_{t y} q_{t v}$ where $p_{i j}$ is the usual transition probability. The probability that the patient makes a transition to state $s_{11}$ is defined as the product of probabilities $p_{\imath}\left(1-q_{w}\right)$. Suppose instead that the patient is not in state $s_{i 7}$ for $i=1,2,3,4$. Then the probability that the patient makes a transition from state $s_{t z}$ to state $s_{1 v}$ or from state $s_{t k}(k=1,2,3,4,5,6)$ to state $s_{j v}$ is defined as the product of probabilities $p_{\imath} p_{a v}$. An example is displayed in Fig. 7 for the transition from state $s_{24}$ ( $q$ is one of the elements of the set $G=\{v, 1$, $2 \ldots, 7\})$ on day $t_{k}$ to state $s_{3 r}(r$ an element of set $G$ ) on day $t_{k+1}$. Note that the probability $p_{23}$ needed to compute the product $p_{23} q_{2 v}$ is obtained directly from the least squares matrix $\mathscr{P}$ in the model of depression.

Unlike the model of depression, only one step is needed to estimate the 8 parameters of the model of utilization. Let $S$ be the 35 by 35 matrix of transition probabilities which define the model of utilization (note that the majority of cells contain structural zeros). Assume that the first row contains the transition probabilities from state $s_{1 v}$ to states $s_{1 v}, s_{11}, \ldots, s_{17}, s_{2 v}$, $s_{21}, \ldots, s_{27}, \ldots, s_{47}, s_{5}, s_{6}$ and $s_{7}$; that the second row contains the transition probabilities from state $s_{11}$ to the aforementioned states: and so on. Let $R$ be the 33 by 33 submatrix which is obtained by omitting states $s_{6}$ and $s_{7}$. 


\begin{tabular}{|c|c|c|c|c|c|c|c|c|c|}
\hline & & \multicolumn{8}{|c|}{ State On Day $t_{k+1}$} \\
\hline & & $s_{3 v}$ & $\mathrm{~s}_{31}$ & ${ }^{s_{32}}$ & $s_{33}$ & $\mathbf{s}_{34}$ & $\mathbf{s}_{35}$ & $s_{36}$ & $s_{37}$ \\
\hline \multirow[b]{3}{*}{ State } & $s_{2 v}$ & $p$ & 0 & $p^{\prime}$ & 0 & 0 & 0 & 0 & 0 \\
\hline & $s_{21}$ & $p$ & 0 & $p^{\prime}$ & 0 & 0 & 0 & 0 & 0 \\
\hline & $\mathrm{s}_{22}$ & $\mathrm{p}$ & 0 & 0 & $p^{\prime}$ & 0 & 0 & 0 & 0 \\
\hline on & $s_{23}$ & p & 0 & 0 & 0 & $p^{\prime}$ & 0 & 0 & 0 \\
\hline Day & $\mathbf{s}_{24}$ & p & 0 & 0 & 0 & 0 & $\mathrm{p}^{\prime}$ & 0 & 0 \\
\hline \multirow[t]{3}{*}{$t_{k}$} & $s_{25}$ & $p$ & 0 & 0 & 0 & 0 & 0 & $p^{\prime}$ & 0 \\
\hline & $s_{26}$ & p & 0 & 0 & 0 & 0 & 0 & 0 & $p^{\prime}$ \\
\hline & $s_{27}$ & $q$ & $q^{\prime}$ & 0 & 0 & 0 & 0 & 0 & 0 \\
\hline $\begin{array}{l}\mathrm{q}^{\prime}=\mathrm{P}_{23}( \\
\text { model of } \\
\text { paramete }\end{array}$ & $p=p$ & $\begin{array}{l}P_{2 v} \\
\text { ere } \\
\text { ion }\end{array}$ & $\begin{array}{l}\text { nd } p^{\prime} \\
23 \text { as } \\
\text { nd wh }\end{array}$ & $\begin{array}{l}\mathrm{P}_{23} \\
\text { the } \\
\text { re } \\
\text { stat }\end{array}$ & $\begin{array}{l}2 v^{\prime} \\
\text { nsit } \\
\text { and } \\
2 \text { wh }\end{array}$ & $\begin{array}{l}\text { hile } \\
\text { on pr } \\
20 \text { ar } \\
\text { ch re }\end{array}$ & $\begin{array}{l}=p_{23}{ }^{g} \\
b_{1} b_{11} \\
\text { the }\end{array}$ & $\begin{array}{l}\text { ond } \\
\text { ty } \mathrm{Er} \\
\text { wo }\end{array}$ & the \\
\hline
\end{tabular}

Fig. 7. Parameters in model of utilization.

Then the matrix $R$ can be used to predict the number of visits a patient makes as an outpatient in states $s_{1 v}, s_{2 v}$ $s_{3,}$ and $s_{40}$ before leaving the system for either states $s_{6}$ or $s_{7}$. If the matrix $N$ is set equal to $(I-R)^{-1}$, then the sum of selected elements within the $i$ th row of $N$ (elements in columns 1,9,17 and 25; also see discussion of matrix $S$ later in the paper) gives the number of outpatient visits made by the patient before entering states $s_{6}$ or $s_{7}$, given that the patient is first observed in state $s_{\text {, }}$ (see [27] for a discussion of the derivation of the fundamental matrix $N)$.

Again, the Becker [26] program was used to obtain the least squares estimates of the parameters. Specifically,

$$
\begin{aligned}
\min (\mathscr{Y}) & =\min \left[\sum_{t \in C}\left(\left(\sum_{, \in C} E_{1}^{\prime} \mathcal{N} E_{1}\right)-v_{t}\right)^{2}\right], \\
C & =\{1,9,17,25\}
\end{aligned}
$$

where $v_{1}$ is the observed number of patients starting in state $s_{t v}$ and making exactly $v_{t}$ visits before being absorbed in either states $s_{6}$ or $s_{7}$.

\section{Results}

Estimates of the parameters $p_{i v}$ and $q_{i v}$ were as expected, i.e. the probability of visiting a psychiatrist on the very next day was much higher for the very depressed patients than for the moderately depressed patients. An indication of the overall fit of the model of utilization can easily be obtained. The predicted number of visits from states $s_{1 v}, s_{2 v}, s_{3 v}$ and $s_{4 v}$ to states $s_{6}$ or $s_{7}$ was, respectively, 20.9, 7.3, 14.6 and 17.1 while the corresponding observed number of visits was $15.1,24.8,15.4$ and 17.5. The agreement is good to a first order approximation except for the predicted number of visits from state $s_{2}$ to states $s_{6}$ or $s_{7}(7.3$ predicted visits as compared with 24.8 observed visits). This may indicate a violation of the assumptions of the model of utilization. However, the observed number is based on very few patients (5) and may therefore be rather less than reliable.

\section{Discussion}

The model of utilization has been developed to the point where it can be used to predict the number of patients making a visit to a psychiatrist in state $s_{w}$ on day $t_{k}$, given knowledge of the number of patients in each state $s_{t w}$ on the day they enter the system (this follows from eqn (1) and the fact that estimates of all the parameters of the full 35 by 35 matrix $S$ of transition probabilities have now been obtained). It remains to estimate the utilization of psychiatric services on day $t_{k}$ for patients in states $s_{1}$ through $s_{4 v}$ and state $s_{5}$. Note that in this context the utilization of psychiatric services by patients in state $s_{1}$ on day $t_{k}$ will be defined as the expected number of physician hours that will need to be devoted to patients in state $s$, on day $t_{k}$, given a particular history of patient arrivals on days $t_{0}$ through $t_{k}$.

This estimate, $U(k)$, can be obtained quite simply from the matrix $S$ and two other vectors, $\underline{H}$ and $Z(k)$, which will be defined shortly. First however it will be convenient to relabel the states of $S$. The states in $S$ which are important from the standpoint of personnel utilization are states $s_{1 v}$ through $s_{4 v}$ and state $s_{5}$. If states $s_{1 v}$ and $s_{11}$ through $s_{17}$ are relabelled, respectively, $u_{1}$ through $u_{8}$, states $s_{2 v}$ through $s_{27}$ as $u_{9}$ through $u_{16}$, states $s_{3 v}$ through $s_{37}$ as $u_{17}$ through $u_{24}$, and states $s_{4 v}$ through $s_{47}$ as $u_{25}$ through $u_{32}$ and if states $s_{5}, s_{6}$ and $s_{7}$ are relabelled, respectively, $u_{33}, u_{34}$ and $u_{35}$, then the 
states which are important from the standpoint of personnel utilization are now $u_{1}, u_{9}, u_{17}, u_{25}$ and $u_{33}$.

Let the vector $\underline{H}$ contain entries $h_{1}$ which represent the time required to meet the needs of a patient in state $u_{i}$. By definition the estimates $h_{i}$ will be positive for $i=1$, 9, 17, 25, 33 and zero otherwise. These estimates can presumably be obtained from patients' charts. Let the vector $Z(k)$ for $k$ greater than zero consist of entries $z_{t}(k)$ which indicate the number of patients one expects to find entering the system in state $u_{1}$ on day $t_{k}$. Again, $z_{t}(k)$ will be nonnegative for $i=1,9,17,25,33$ and zero otherwise since knowledge about a patient is not available until the patient makes a visit to the system. These estimates will presumably reflect the demographic characteristics of a particular health planning locale.

The estimate $U(k)$ of the utilization of psychiatric services on day $t_{h}$ can now be written as a function of $S$, $\underline{H}$ and $\underline{Z}(k)$ :

$$
U(k)=\sum_{j=0}^{h} \underline{Z^{\prime}}(j) S^{h}{ }^{\prime} \underline{H}
$$

where $Z(0)$ gives the number of patients currently in each of states $u_{1}$ through $u_{33}$ and where $S^{0}$ is defined as the identity matrix.

Two final remarks about the model of utilization should be made. First, it was claimed in the beginning of the paper that linking the model of utilization to a model of the disease process had at least one potential advantage. The advantage comes from the fact that changes in the disease process are automatically reflected in changes in the level of utilization. For example, suppose that one hypothesized or had evidence that the first order transition probabilities $p_{31}$ and $p_{43}$ were increased by some amount due to a new therapeutic treatment. Then, one can immediately determine the effect on utilization by changing $p_{31}$ and $p_{43}$ in matrix $P$ to their new settings and calculating $U(k)$ under the new settings.

Second, it was claimed in the beginning of the paper that the model could incorporate at least some of the prevailing economic factors. One factor that would clearly seem to be influenced by the state of the economy is the frequency of visits. As funds become more scarce, presumably patients will have to cut back on visits. On might expect physicians in such a situation to handle the decrease by spacing out the visits of the least depressed patients while maintaining at the optimum spacing the visits of the most depressed patients. The influence of such changes in visit frequency can be simulated quite easily with the models discussed above. For example in the current hypothetical situation one might decrease the probabilities $q_{1 \text { t }}$ and $q_{2 v}$ that a patient in, respectively, states $s_{17}$ or $s_{27}$ on day $t_{r}$ visits a therapist on the very next day $t_{r+1}$ while keeping constant the probabilities $q_{3}$, and $q_{4 v}$.

\section{CONCLUSION}

The estimation of the utilization of inpatient and outpatient psychiatric services presents special problems. One way of dealing with the problems is first to construct a model of a disease and only then to construct a model of utilization. Such was the approach followed in the current paper.

The approach looks promising. The various predictions of the models are in good agreement with the observations. Furthermore, estimates of the model parameters could be obtained from data which in most cases already are readily available or could easily be made so. Finally, the models can be used to predict such quantities of interest as the incidence, prevalence and recovery rates. In addition, the models can predict the consequences for utilization of changes in the treatment regimen.

The major weaknesses of the approach are more or less self-evident. The concentration on a disease-specific model of utilization leaves open the question of whether the approach would work for other diseases. And the failure to incorporate the influence of changing economic factors on the level of utilization means that one must be very careful about extrapolations more than several years into the future.

The above criticisms notwithstanding, the current paper suggests an approach to the prediction of the utilization of psychiatric services which is itself new and which can potentially be generalized to other diseases and areas of application.

Acknowledgements-Major portions of this research were supported by a grant from the National Institute of Mental Health (MH16413) to Dr. David Knesper and by a grant from the Michigan Department of Mental Health to Donald Fisher. The authors wish to thank Dr. John G. Cross and Dr. Gardner C. Quarton for their helpful comments and criticisms throughout the course of the study. Special thanks go to Susan Duffy. She suggested several of the procedures which are described in the paper and used to estimate various of the model parameters. Requests for reprints can be sent either to David Knesper or Donald Fisher Fisher is now at the University of Massachusetts. 114 Marston Hall, Amherst, MA 01003. U.S.A

\section{REFERENCES}

1. J. R. Lave, L. B. Lave and S. Leinhardt, Medical manpower models: need, demand and supply. Inquiry 2, 97-125 (1975).

2. B. E. Erlich and J. M. Diamond, Lithium, membranes and manic-depressive illness. J Membrane Biology 52, 187-200 (1980).

3. E. P. C. Kao and G. G. Tung, Forecasting demands for inpatient services in a large public health care delivery system Socio-Econ. Plan. Sci. 14, 97-106 (1980).

4. E. P. C. Kao and G. G. Tung, Aggregate nursing requirement planning in a public health care delivery system. SocioEcon. Plan. Sci 15, 119-127 (1981)

5. E. P. C. Kao, A semi-Markov model to predict recovery progress of coronary patients. Health Services Res., 191-208. (1972).

6. W. G. Sullivan and E. L. Blair, Predicting workload requirements for scheduled health care services, with an application to radiology departments. Socio-Econ. Plan. Sci 13. (1979).

7. J. W. Bush, M. M. Chen and J. Zemba, Estimatıng health program outcomes using Markovian equilibrium analysis of disease control. Am. J. Public Health 61, 2362-2375 (1971).

8. V. Navarro, A systems approach to health planning. Health Services Res. 4, 96-111 (1969).

9. V. Navarro, Planning personal health services: A Markovian model. Medical Care 3, 242-249 (1969).

10. E. Schach and S. Schach, A continuous time stochastic model for the utilization of health services. Socio-Econ Plan. Sci 6, 263-272 (1972).

11. R. W. Chorba and J. L. Sanders, Planning models for tuberculosis control programs. Health Services Res 6. 144-164 (1971).

12. R. Davies, D. Johnson and S. Farrow, Planning patient care with a Markov model. Op. Res Quart. 26, 599-607 (1975)

13. J. K. Fried, M. Marcus and A. B. Forsythe, Markovian model for evaluating dental-care programs. Community Dentistry and Oral Epidemiology 7, 25-29 (1979). 
14. J. R. Newheiser and M. E. F. Schoeman, A stochastic model for health care resource planning. Socio-Econ. Plan. Sci. 6 , 197-213 (1972).

15. J. Ortiz and R. Parker, A birth-life-death model for the planning and evaluating of health service programs. Health Services Res., 6, 120-143 (1971).

16. W. H. Thomas, A model for predicting recovery progress of coronary patients. Health Services Res., 185-213 (1968).

17. G. O. Ware and P. M. Dickert, A management planning model for the delwery of family planning services. SocioEcon. Plan. Sci, 10, 155-158 (1976).

18. A. Sweillam and K. Tardiff, Prediction of psychiatric inpatient utilization: A Markov chain model. Administration in Mental Health 6. (1978).

19. L. G. Kessler, D. M. Steinwachs and J. R. Hankin, Episudes of psychiatric utilization. Medical Care 18, 1219-1227 (1980).

20. I. Moscovice, A method for analyzing resource use in amhulatory care settings. Medical Care 15, 1024 (1977).

21. J. A. Solon. J. J. Feeney, S. H. Jones, R. D. Rigg and C. G. Sheps, Delineating episodes of medical care. Am. J. Public Health 57, 401 (1967)

22. D. M. Steinwachs and R. Yaffee, An approach to the review of utilization and quality of ambulatory care. Paper Presented at the Op. Res. Soc. Am. (ORSA) Meeting, Puerto Rico, 1974.

23. G. Dunn and D. Skuse, The natural history of depression in general practice: stochastic models. Psychological Medicine 11, 755-764 (1981).

24. F. N. Johnson and S. Johnson, Lithium In Medical Practice. University Park Press (1978).

25. B. Carroll J. M. Fielding and T. G. Blashki, Depression rating scales: a critical review. Arch. General Psychiatry 28 , 361-366 (1973).

26. John R. Becker, EXPLORE: A Computer Code for Solving Nonlinear, Continuous Optimization Procedures. Aun Arbur, Michigan: Division of Research, Graduate School of Business Administration (Jan. 1974).

27. J. C. Kemeny and I. Snell, Finite Markov Chains. Van Nostrand, New York (1960)

\section{APPENDIX A}

Given the matrix $P$ of transition probabilities forming an ergodic Markov chain. Kemeny and Snell[27] show how to obtain the matrix $F$ of expected first passage times. In the current context the matrix $P$ is found as follows. First, the transition probabilities from states $s_{6}$ to $s_{6}$ and from state $s_{7}$ to $s_{7}$ (see cells labelled 19 in Fig. 1) were set equal to zero and the transition probabilities from state $s_{6}$ to $s_{1}$ and state $s_{7}$ to $s_{1}$ were set equal to one.

Second, the entries in row $i$ of the matrix $Q$ are multiplied by a constant such that:

$$
\begin{aligned}
& \sum_{i=1}^{4} q_{1,} r_{1}=1-p_{17} ; 0 \leq p_{17} \leq 1 \\
& \sum_{j=1}^{4} q_{3} r_{1}^{\prime}=1-p_{16} ; 0 \leq p_{26} \leq 1 ; i=2,3,4,5
\end{aligned}
$$

The entry $p_{1,}(j=1,2,3,4)$ in matrix $P$ is set equal to $q_{1}, r_{t}$ and the entries $p_{t i}(i=2,3,4,5 ; j=1,2,3,4)$ in matrix $p$ are set equal to the respective entries $q_{i j} r_{1}^{\prime}$. This fills in cells $1-14$ in Fig. 1. Note that this manipulation assures that the rows sum to 1 in the matrix $P$.

Finally, the estimates of cells 15,16 , and 17 are taken directly from Fig. 2.

\section{APPENDIX B}

It needs to be shown that the procedure outlined in step 1 cannot be used to derive the probabilities $p_{16}$ and $p_{17}(i=1,2,3$, 4). An example can best illustrate the problem. Suppose a patient was in the system 999 days. Suppose the patient starts and leaves in state $s_{1}$. Suppose 100 transitions are made from state $s_{1}$ to state $s_{1}$ and 90 transitions are made from state $s_{1}$ to state $s_{2}$. Finally suppose that only 10 transitions from state $s_{1}$ to state $s_{1}$ are observed (i.e. recorded by the psychiatrist) and that only 9 transitions from state $s_{1}$ to $s_{2}$ are observed. Then, using the procedures of step 1 it follows that $q_{11}=10 / 20, q_{12}=9 / 20$ and $q_{17}=1 / 20$. But clearly $q_{17}$ is an overestimate since if all transitions were recorded one would find that $p_{17}=1 / 200$.

However, note that the estimates of the probabilities $q_{11}$ and $q_{12}$ will correctly reflect $p_{11}$ and $p_{12}$ if transitions from state $s_{1}$ to state $s_{7}$ are not included. That is, ignoring this transition, one obtains $q_{11}=10 / 19$ and $q_{12}=9 / 19$ for the probabilities computed using the observations taken on only those days visits were obtained while one obtains $p_{13}=100 / 190$ and $p_{12}=90 / 190$ for underlying transitions.

The reader might argue that the above example was "rigged" in the sense that the proportion (0.1) of observed transitions from state $s_{1}$ to $s_{1}(10)$ and from state $s_{1}$ to $s_{2}(9)$ was kept constant, i.e. $10=0.1(100)$ and $9=0.1(90)$. However, it can easily be shown that the assumptions stated in the text (step 1) lead to constant proportions. This is left as an exercise for the reader. 